\title{
CLIMATE CHANGE AND THE ECOLOGICAL PSYCHOLOGY
}

Viktor I. Panov,

Shamil R. Khisambeyev

Psychological Institute of Russian Academy of Education

\begin{abstract}
Psychological effects of climate change constitute one of the subjects of study of ecological psychology. Ecological psychology is formed and developed at the junction of ecology, different directions of psychology, psychotherapy, pedagogy, philosophy and other disciplines. The article is devoted to review those areas of psychological research, which consider the human psyche in the logic of interaction with the environment. As a result, we hope to answer the fundamental question of whether ecological psychology may be regarded as an independent area of psychological research, which has its object and methods that distinguish it from other areas of psychological theory and practice.
\end{abstract}

Keywords: ecological psychology, mental process, extreme psychology, psychology of global change, system "human-environment".

The words "ecology" and "psychology" are of Greek origin, and the father of medicine Hippocrates in ancient Greece was the first who formulated the idea of communication of the human condition, including its spiritual qualities, and the environment.

Ecological psychology is a definite trend of psychological science that has been formed in Europe and the U.S.A. in the sixties years of the last century and are grouped under the general name for at least three areas of psychological research: psychological ecology, environmental psychology and an ecological approach to perception. Currently, different departments conduct studies in ecopsychology, but apart from one another, and using different theoretical foundations and methods.

Kurt Pawlik, leader of the largest international program on ecological psychology at the end of the last century defines ecological psychology (or: ecopsychology) as study of mental processes in the environmen- 
tal conditions under which the experience and the behavior taking place "in themselves" without interference from the researcher. The ecological psychology studies human experience and behavior in close connection with the environment and attempts to give them a theoretical justification.

The starting point for eco-psychological research is the notion that mental processes, states and consciousness, mental development, learning and behavior, as well as mental health can not be viewed in isolation, a particular individual with his environment and nature in general.

One of the most significant characteristics of the environment is the climate. It affects the psyche in three ways:

- the first - directly, through physiological processes;

- the second - indirectly through effects on agriculture, trade, tourism and other activities;

- the third - indirectly through the submission, installation, imperatives, being introduced by the media.

Let us try to review those areas of psychological research, which consider the human psyche in the logic of interaction with the environment. As a result, we hope to answer the fundamental question of whether ecological psychology may be regarded as an independent area of psychological research, which has its object and methods that distinguish it from other areas of psychological theory and practice.

\section{Environmental problems}

Environmental issues in determining the relevance and interest in ecological psychology at the present stage of its development, is the realization that the preservation of the environment on the planet and the planet as a whole ecosystem is impossible without changes in human's relationship to the natural environment and to the nature of its own.

In its development, the mankind had possessed powers which allow him to destroy the system of regulation of the environment. The ecological crisis - the discrepancy between the level of impact on nature and those boundaries, which should be the impact to the system of life could adequately regulate the environment. Therefore, the main work is modification of human consciousness. 
According to analysis by the philosopher Vittorio Hösle, the main trends of development of mankind in the last century, were determined by the economic paradigm, orienting the human consciousness to the consumer attitude toward the world of nature around, to other people, and to himself.

It is the dominant influence of the economic paradigm of human development has caused the technocratic nature of our civilization. And this in turn led to the fact that human consciousness was formed predominantly in the context of the transformation of real properties and relations of the world in order to use them for survival and economic well-being. And therefore, as pointed out by Hösle, nature until the XX century remained without rights, that defines the relationship to her as an object of exploitation and consumption of its resources.

One consequence of the economic paradigm has become climate change, keenly felt in those regions where the ecological balance is very fragile.

Overcoming the ecological crisis can not be accomplished within the technocratic way of thinking prevailing in the public and individual consciousness of people in developed countries. It is required reorientation of human consciousness on the need to preserve the planet and its resources. A necessary condition for such a change of consciousness of people is to adopt the environmental imperative as a guide to action. Under the environmental imperative is understood such interaction with nature, according to which there is only one right and allowed way - that does not disturb the existing ecological balance in nature.

According to the ideas of Vernadsky, the problem of preserving the environment can not be achieved until mankind realizes that it is both a product of nature and a resource of the development of system "human - the planet". The latter means that a person should be regarded as a subject, which implements the process of self-realization his ability to live the universal principles of self-nature, including human and planet as a whole.

Awareness and acceptance of human responsibility for his own development itself, the planet and nature in general means that a person becomes subject to ecology, i.e. subject to ecological process of development. 
However, it is easy to see that questions of formation of human consciousness and the use of its capacity for self-development in the interaction with the environment - it is the scope of psychological research. Therefore, there is the problem of studying the ecological consciousness, as well as the development of methods of diagnosis and formation of ecological consciousness among professionals and the general population.

\section{The problems of extreme psychology}

The instability of socio-economic and political conditions of life, increasing the number of natural and man-made disasters, crime, insecurity, lack of psychological preparation for life in extreme environments - all of these traits with particular acuteness pose practical problems of psychological preparation and readiness for life in complex, stressful conditions, as well as psychological assistance to children and adults in extreme situations. The crisis alters mental status and behavior of people in extreme conditions, and the psychological support of combatants and search and rescue operations, their families and affected communities is required, as well as their subsequent social and psychological rehabilitation. It is about learning and developing methods for the use of natural, social, and virtual-environmental factors for the practical changes in mental states of people in everyday, and extreme and post-extreme conditions as a means of psychological rehabilitation.

\section{Psychological ecology}

This direction is in fact one of the branches of human ecology and has been studying the effects on the human psyche environmental factors of chemical and physical nature. These include, for example, air and water pollution, increased background radiation, etc.

As examples are numerous studies on the effect of elevated radiation levels in the area of the Chernobyl accident and many other studies of this kind.

The presence of a real impact of environmental factors, a dose which exceeds the acceptable norm for a given individual, changes his physiological and as a consequence, the mental state. In addition to purely 
medical symptoms, altered mental state under the influence of these environmental factors can be manifested in increased aggressive behavior, a general decrease in vitality (mental activity, attention, etc.) in the emergence and development of depression, etc.

In addition to the environmental standards of permissible impact of physical, chemical, and similar factors should be considered, firstly, the individual and the age range of the sensitivity of the organism and psyche to such influences. In other words, the same dose of exposure may be critical for one individual and noncritical for another. And, secondly, it should be born in mind that the impact of this kind can be accumulated, i.e. receiving individual small doses of non-hazardous, but permanent and long-term, may lead to the same effect as a one-time impact extremely high doses of the same exposure.

Psychogenic influence of physico-chemical factors of the environment may depend not only on the value of their parameters, but also on the attitudes of the people. For example, Claude Levy-Leboyer and Yves Duron argue that awareness of uncontrollability of environment events is a source of helplessness, lead to the development of external locus of control and reduce feelings effectiveness of their own activities.

It is important here to note that the physical and chemical properties of the environment are, of course, physical, chemical or biological nature of the effect because they affect the person on the physico-chemical level of its interaction with the environment. Whereas changes in mentality in this case caused a corresponding change in the organic structures and systems of the human body, i.e. are secondary to these changes in the human body.

\section{The psychology of global change}

A special kind of environment as an object of perception and learning are the so-called global changes in natural conditions of human habitation in large parts of regional and planetary scale. Their study is the subject of psychology of global change.

The term "global change" is used to refer to such problems of the environment that are associated with global warming, tropical deforestation, etc. Global changes are a consequence not only of natural forces, as in the case of natural disasters as a result of human activity. It is im- 
possible to avoid the effects of global change, moving, for example, in unpolluted place.

Experts note the following distinctive features of the phenomena attributed to "global change":

- They are characterized by large spatial and temporal scales and caused directly by human activities, acquiring planet scales;

- They are irreversible, and they have such a low "signal-noise" ratio and occur so slowly that human consciousness has no mechanism for direct perception of the process of generating a catastrophic situation, until the very catastrophe becomes inevitable and does not take a clear, visible character (for example, rise in water level, drought and etc.);

- They are characterized by a high degree of masking and delayed causation;

- They are characterized by large spatial and social distance between the agents and victims of global change;

- Information about them that lacked clarity, so experts on global change could not come to an agreement on the extent and timing of the real threat;

- The mass consciousness of people tend to ignore information about the threat to their safety due to global change.

As the most urgent problems of psychology of global change are marked the following:

- The need to create a professional and adequate perception of the speed of the global changes, as well as an adequate risk assessment of these changes to human life and ecological balance on the planet;

- The need to change the behavior of those who are responsible for the occurrence and prevention of adverse global changes.

Thus as a matter of psychology of global change are such ecologically significant changes in the environment that occur outside the functional range of direct perception, evolutionary prevailing in humans.

In addition to these studies of global changes, there are several studies on the perception of the natural micro-environment, i.e., a collection of objects animate and inanimate nature, constituting the surrounding landscape. The results of these studies are summarized by psychologists as the following provisions: 
- The natural environment has no specific, firmly fixed in time and space boundaries. The idea is that allocation and structuring of boundaries immediately perceived by the human environment is relative, subjective colored character. For example, a fisherman, a geologist or an artist perceive the same landscape and its objects in their own way.

However, the psychological interaction between humans and the natural environment may have the opposite direction, as the different degree of structuring of the landscape may have a psychogenic influence on the individual. And there are like two poles here. The first example is the chaos of the rainforest, which cause mental fatigue. The opposite example is the "empty" environment - it's the monotony of space, not giving any visual or other landmarks perceiving individual. In some people, the natural environment of this type causes a feeling of causeless anxiety and even fear: the white silence of the tundra, endless deserts, etc.

- The environment simultaneously affects all the organs of senses. This means that the perception of the environment has over-modality character. It includes not only the visual perception, but also the perception of sounds (such as birds singing or the wind noise), perception of odors, etc. Therefore, the perception of the environment (it is sometimes still referred to as the perception of "Field") differs significantly from the perception in the laboratory, which is characterized, even as it must be an artificial restriction conditions and modalities for the perception of objects in accordance with the objectives of the study.

- Perception of the environment due to the nature of the subject of perception. In this case we are talking about the fact that the same properties of the environment (including the boundaries of what was said) are perceived by a person not in the abstract, but functional - depending on the type of activity and for which the individual is in the nature environment. The same pine grove to the woodcutter, an ecologist or the mushroom picker as an object of perception appears in different forms.

- Each element of the natural environment along with the physical and chemical characteristics has a social value. This feature of the perception of the environment is closely linked to the previous one, because the social nature of man is also manifested in the social condition of its perception. Features of perception of the environment may be due to the historical value of the landscape (eg, the perception of the field of Borodino), ethnic traditions ("Japanese garden"), etc. It is important to 
note that the socio-cultural importance of the environment as an object of perception, on the one hand, is formed in the course of human socialization, and on the other - it serves as a means of their socialization. Examples are the images of the natural environment, presented in artistic and ethnic traditions of different peoples.

- The perception of the natural environment is characterized by integrity. This is a very important feature, because the natural environment is perceived not as a fragmented set of spatial and other properties and relations, but as a whole, though composed of differing parts. Despite the limitations noted above the selectivity of perceptual features and objects of natural environment, it nevertheless always perceived as a gestalt. This determines the perception of the integrity of its individual parts.

The foregoing features of perception of the environment, despite some evidence of interest in understanding the environment as a component of the relation "human-environment". Namely, the environment as an object of perception is relative, since the boundaries, the amount and structuring of its spatial, object and other properties depend largely on the subjective characteristics of the observer.

\section{The environmental psychology}

To the 60-th years of XX century among psychologists increasingly have begun to argue the realization that "laboratory" psychology does not and can not give a valid representation of the psychological characteristics and patterns of human behavior "in the real world". The result of such mentality the first international research program on environmental psychology was adopted in 1982 in Edinburgh.

The main directions of the field of psychological research have been recognized the following:

- environmental cognition,

- environmental behaviour,

- environmental assessment,

- environmental stress.

As a subject of study in the psychology of the environment are the relation "human-environment", that is the relationship between environmental variables and the different psychological characteristics of human behavior. 
Methodologically environmental psychology characterized by the following:

- people and the environment are treated as components of a unified system. This means that the initial basis for determining the research subject is the system "human-environment";

- as a source is accepted idea that the environment is so important influences on human behavior, that in the reactions of different individuals on exposure to the same environment is found more in common than different.

Describing the current state of the environmental psychology, Daniel Stokols notes that the direction of applied research and theory development in this area are considered in light of the different natural and social disasters, including global environmental change, the spread of violence in regional and international levels, the impact of new information technologies on profession and family life, raising the cost of health care services, and social processes of aging. The result is that those engaged in research psychologists are becoming more and more interested in finding the "core" of context and directly environmental research.

In other words, the state of environmental psychology is characterized by the same conceptual diversity which makes it necessary to define a single object and subject of this research, which is typical for domestic eco-psychological research.

Having for its object the psychological aspects of human interaction with its environment, it is, in essence, brings together three areas differing nature of the interaction in the system "human-environment":

- psychology of environmental influences, which are the subject of study and practice effects on the human psyche of the environment of different modalities - both natural and social: urban or architectural space, family and educational environment, etc.;

- extreme psychology, the subject of study which are the psychological characteristics of mental states, behavior, and human activities in emergencies (natural and manmade disasters, terrorist acts, etc.) and unusual conditions for a man (under water, in air in space, underground, etc.);

- psychology of environmental protection, the subject of which are psychological aspects of reasonable and intact relationships to 
the natural resources of the planet and to the social and cultural achievements of the mankind, responsible attitude to waste from human activities that pollute the environment, etc.).

\section{The psychology of the information environment}

So-called information environment is kind of social environment, which formed a set:

- Subjects of information interactions or influences;

- The actual information for use by the subjects of the information sphere;

- Information infrastructure, providing an opportunity for exchanging information between the actors;

- Public relations developing in connection with the formation, transmission, distribution and storage of information.

From a psychological point of view information environment is presented in the form of information influences of various kinds, which a person are currently experiencing, as well as in the form of communication, interaction with others and the impact on others (media, multimedia, Internet, etc.). A distinctive feature of this type of environment is that in most cases it is used as a "psychological instrument" (according to Vygotsky) of social impacts on humans.

As such, the information environment is a critical factor in the socialization of the individual, as well as the formation of individual and social consciousness. It serves as the means by which a society transmits norms, values and patterns of behavior to individuals, namely it has a direct influence on the social behavior.

However, the increasing pace of informatization of society exacerbates ecopsychological problems, because a technocratic approach to information society poses a threat comparable to the scale of the threat of nuclear and environmental disasters. The objective of this direction is to provide psychological security of the main interests of individuals, society and state from the threats associated with exposure to the information environment on the psyche of citizens.

One of the most common types of information medium is television. Television as a means of communication is fundamentally different from all others. If the word always refers to the concept and thus implies 
a certain abstraction, the picture just shows the thing. Image originally less cognitively loaded than the word: the word requires a recognition of its true value, while the image appeals more to emotions than to reason. It asks us to feel rather than think.

All the researchers emphasize that the greatest influence television has on young people when there is a rapid process of socialization, and impressionable viewers not only identify themselves with the heroes of transmission, but also transfer the borrowed patterns of behavior in real life.

The examined directions of ecopsychological studies differ from each other not only in methods but also the subject of investigation. In some cases, as such serve mental processes (e.g. perception), mental states (e.g. critical), ecological consciousness and human behavior, in other cases psychogenic properties of the environment: spatial, educational, informational, etc.

Nevertheless, a common feature of these trends is that as the initial basis for determining the object and the subject of environmental and psychological research is used system relation "human-environment".

At the same time in different areas of ecological and psychological studies have used the description of the environment, built on different grounds. As such, you can specify:

- substantive principle, that cause a description of different kinds of environment, whose properties are initially specified and known: the natural environment (physical-chemical and biological), spatial (both natural and anthropogenic), social environment (family, educational, informational and ethnocultural), life environment and so on;

- ecological principle, when the environment is regarded as a habitat that provides the opportunity or obstructive for satisfaction of vital human needs. In this case, the starting point for descriptions of the environment is the isolation and description of its biological, social, spiritual and other needs;

- the principle of activity according which the environment appears as a space of possibilities for handling the various activities: game, education, sports, art, etc.;

- functional principle, when different kinds of environment are considered to start from the functional role it plays as a component of the system "human-environment". 
There are three significant differences between the psychological ecology and environmental psychology.

1. In the psychological ecology of the impact of environmental factors are treated as separate variables, while the psychology of environmental impact of environmental factors is considered as a system, if possible, taking into account all factors. For example, such spatial factors as crowding of people or closure of premises, are in the psychological environment are not considered.

2. In the psychological ecology environmental factors have not psychological nature, whereas in the psychology of the environment, more precisely in the part, which we designated as the psychology of environmental influences, they are psychologically conditioned nature. Such environmental factors as "aggressive" television movie, "funny" advertisement or "psychological tension" of learning situations are subjective. The subjectivity is generated by people who use the object as the subjects of the characteristics of the environment (e.g. television screen) as a means for people to achieve their creative, promotional, educational or other purposes. But because this subjectivity is expressed not in the direct action (for example, the same television director), but indirectly - through the object properties of the TV screen, in this case it makes sense to talk about environmental impact.

3. The environmental psychology studies the scientific and practical aspects (research, design, assessment, rehabilitation) of such environmental factors (natural, human, spatial, social, educational, informational and other) which effect on the human psyche. This means that, in contrast to the psychological environment, it is about the impact on the human psyche (the cognitive processes and emotional experiences, personality traits and behaviors) such properties of the environment that have the character materialized psychological attributions and in this sense can be considered as having psychological nature.

In conclusion, we would hope that the cooperation of scientists dealing with various aspects of environmental influences on a person, including climate change, will adequately meet the challenges of time and will give future generations a chance to a more harmonious life. 